\title{
Io's Volcanos: Latest Results from Galileo and from the Earth
}

\author{
John Spencer
}

Lowell Observatory, 1400 W. Mars Hill Rd., Flagstaff AZ 86001, USA

\begin{abstract}
Three recent close flybys of Io by the Galileo spacecraft, and new observations from the Hubble Space Telescope and ground-based telescopes, have greatly advanced our understanding of Jupiter's volcanic moon Io. Io's volcanos are much hotter than previously suspected, perhaps requiring exotic silicate magma compositions. Despite much new data, Io's largest volcano, Loki, is still poorly understood. New data on Io's plumes suggest the existence of two types of plumes: primary plumes, relatively rich in $S_{2}$ gas, which are emitted where magma first reaches the surface, and secondary plumes, more $\mathrm{SO}_{2}$ rich, which result from interaction of lava flows with a volatile-rich substrate.
\end{abstract}

\section{Introduction}

Jupiter's moon Io is one of the most unusual places in the Solar System, due to tidal heating that produces a heat flow near $2.5 \mathrm{~W} / \mathrm{m}^{2}$, roughly 40 times that of the Earth. Io therefore exhibits intense volcanism and rapid resurfacing at rates up to $1 \mathrm{~cm} /$ year. The volcanism has effects beyond Io itself because erupted volatiles escape into the Jovian magnetosphere, producing a dense plasma that erodes and alters the surfaces of Jupiter's other moons, and generates detectable emissions from the radio to the EUV. Here I highlight some of the more notable recent discoveries pertaining to Io's volcanism.

\section{Modeling of Volcanic Emission}

Much of our knowledge of Io's volcanos comes from studying their thermal emission. Models of volcanic thermal emission (Carr 1986; Davies 1996; Howell 1997; Keszthelyi \& McEwen 1997) show that emission from most Io volcanos can be matched using just three parameters, which can thus be constrained observationally. These are: the temperature of the erupted magma, which controls the color temperature at the shortest wavelengths (nearest infrared in the case of silicates); the areal production rate of new magma, which controls the total short-wavelength (near-infrared) flux, and the average age of exposed surfaces, which controls the long-wavelength color temperature. For instance, emission from very dynamic eruptions such as fire fountains is characterized by very short mean ages, measured in minutes even for eruptions lasting hours or days, as hot magma is rapidly exposed, cooled, and buried. 


\section{New Constraints on Volcanic Temperatures: High-Temperature Silicates}

After their discovery by Voyager, observations of Io's volcanos continued using earth-based infrared telescopes. A major outburst in 1986 (Johnson et al. 1986) required temperatures of $1550 \mathrm{~K}$ (Veeder et al. 1994), probably implying silicate volcanism. Carr (1986) and Blaney et al. (1995) argued that the $>5 \mu \mathrm{m}$ volcanic emission seen by Voyager and from Earth, which required temperatures $<400$ $\mathrm{K}$, could also be explained as resulting from old, cool, silicate flows.

Monitoring of the short-wavelength volcanic thermal emission from Earth showed extreme variability (a factor of $\geq 60$ at $2.3 \mu \mathrm{m}$ ), resulting from frequent, brief, high-temperature eruptions consistent with silicate fire-fountaining and/or lava lake activity at temperatures $\geq 1400 \mathrm{~K}$ (Spencer et al. 1997; Stansberry et al. 1997).

Galileo observations have pushed temperatures even higher, to $>1500 \mathrm{~K}$, probably requiring magma temperatures $>1700 \mathrm{~K}$, too hot for basaltic volcanism and perhaps implying more $\mathrm{Fe}$ and $\mathrm{Mg}$-rich, Si-poor, "komatiite" volcanism (McEwen et al. 1998a). Galileo has also directly imaged fire-fountaining, lava lakes, and extensive young lava flows, confirming models derived from earlier, lower-resolution data (McEwen et al. 2000; Lopes-Gautier et al. 2000; Spencer et al. 2000a).

\section{Loki}

Loki is Io's largest volcano, responsible for $10-20 \%$ of Io's total heat flow (Spencer et al. 2000a; Veeder et al. 1994). A wealth of new data on Loki has improved our understanding of its behavior, but underlying mechanisms are still poorly understood. Roughly once per year, Loki's $200-\mathrm{km}$ wide horseshoe-shaped caldera appears to be resurfaced over a period of a few months, leaving vast areas of cooling lava that radiate most of the heat flow (Howell et al. 2000). Temperature mapping of Loki by Galileo shows large temperature variations across the caldera which change during the course of one of these resurfacing events (Spencer et al. 2000a; Lopes-Gautier et al. 2000), but neither of the two most likely resurfacing models, spreading lava flows or overturn of the crust of a caldera-filling lava lake, can easily explain the temperature patterns seen. Another mystery is the faintness of Loki's $<1 \mu \mathrm{m}$ thermal emission (McEwen et al. 1998b), indicating an unusually low magma temperature, or very quiescent eruption conditions that expose little hot magma despite the huge volume of the eruptions.

\section{New Insights into Io's Plumes}

Recent observations have also shed light on Io's plumes, among the most dramatic manifestations of its volcanism. Close-up Galileo images of the Prometheus plume, whose source moved $75 \mathrm{~km}$ between 1979 and 1996, suggest that the plume results from vaporization of volatile surface materials, probably mostly $\mathrm{SO}_{2}$ frost, at the margins of an advancing lava flow (McEwen et al. 2000). Recent Hubble Space Telescope UV spectroscopy has identified $S_{2}$ gas in the Pele plume (Spencer et al. 2000b), in addition to the more abundant $\mathrm{SO}_{2}$ gas previ- 
ously identified at Pele (McGrath et al. 2000). Because $S_{2}$ molecules are likely to polymerize to form reddish $S_{3}$ and $S_{4}$ on Io's surface, this discovery may explain the red deposits found around Pele and, on a smaller scale, near several other Io volcanos. These results confirm the McEwen \& Soderblom (1983) distinction between sulfur-rich "Pele-type" and $\mathrm{SO}_{2}$-rich "Prometheus-type" plumes, except that $\mathrm{SO}_{2}$ also appears to be a major constituent of the Pele-type plumes. Because the red deposits typically occur near calderas, it appears that red plume deposits are from primary plumes, associated with vents where magma first reaches Io's surface and releases $\mathrm{S}_{2}$-rich gas. Prometheus-type plumes appear to be secondary, resulting from mobilization of surface volatiles, and occur at the toes of active lava flows, often some distance from the primary vent.

\section{References}

Blaney, D., Johnson, T. V., Matson, D. L., \& Veeder, G. L. 1985, Icarus, 113, 220

Carr, M. 1986, JGR, 91, 3521

Davies, A. G. 1996, Icarus, 124, 45

Howell, R. R. 1997, Icarus, 127, 394

Howell, R. R. et al. 2000, JGR, submitted

Johnson, T. V., et al. 1988, Science, 242, 1280

Keszthelyi, L., \& McEwen, A. S. 1997, GRL, 24, 2463

Lopes-Gautier, R. et al. 2000, Science, 288, 1201

McEwen, A. S. \& Soderblom, L. 1983, Icarus, 55, 191

McEwen, A. S., et al. 1998a, Science, 281, 87

McEwen, A.S., et al. 1998b, Icarus, 135, 181

McEwen, A.S. et al. 2000, Science, 288, 1193

McGrath, M. A., Belton, M. J. S., \& Spencer, J. R. 2000, Icarus, 146, 476

Spencer, J.R. et al. 1997, GRL, 24, 2451

Spencer, J. R. et al. 2000a, Science, 288, 1198

Spencer, J.R., Jessup, K., McGrath, M.A., Ballester, G.E., \& Yelle, R.V. $2000 \mathrm{~b}$, Science, 288, 1208

Stansberry, J. A., Spencer, J. R., Howell, R. R., Dumas, C., \& Vakil, D. 1997, JGR, 24, 2455

Veeder, G.L., Matson, D.L., Johnson, T.V., Blaney, D.L., \& Goguen, J.D. 1994, JGR, 99, 17095 